University of Massachusetts Amherst

ScholarWorks@UMass Amherst

2003

\title{
Nonnuclear Hyper/Ultraluminous X-Ray Sources in the Starbursting Cartwheel Ring Galaxy
}

Y Gao

QD Wang

University of Massachusetts - Amherst

PN Appleton

RA Lucas

Follow this and additional works at: https://scholarworks.umass.edu/astro_faculty_pubs

Part of the Astrophysics and Astronomy Commons

\section{Recommended Citation}

Gao, Y; Wang, QD; Appleton, PN; and Lucas, RA, "Nonnuclear Hyper/Ultraluminous X-Ray Sources in the Starbursting Cartwheel Ring Galaxy" (2003). The Astrophysical Journal Letters. 1012.

$10.1086 / 379598$ 
Draft Version February 2, 2008

Preprint typeset using LATEX style emulateapj v. 7/15/03

\title{
NON-NUCLEAR HYPER/ULTRALUMINOUS X-RAY SOURCES IN THE STARBURSTING CARTWHEEL RING GALAXY
}

\author{
Yu GaO ${ }^{1}$, Q. Daniel Wang ${ }^{1}$, P. N. Appleton ${ }^{2}$, and Ray A. Lucas ${ }^{3}$ \\ (Accepted 2003 September 5) \\ Draft version February 2, 2008
}

\begin{abstract}
We report the Chandra/ACIS-S detection of more than 20 ultraluminous X-ray sources (ULXs, $L_{0.5-10 \mathrm{keV}} \gtrsim 3 \times 10^{39} \mathrm{ergs} \mathrm{s}^{-1}$ ) in the Cartwheel collisional ring galaxy system, of which over a dozen are located in the outer active star-forming ring. A remarkable hyperluminous X-ray source (HLX, $L_{0.5-10 \mathrm{keV}} \gtrsim 10^{41} \mathrm{ergs} \mathrm{s}^{-1}$ assuming isotropic radiation), which dominates the X-ray emission from the Cartwheel ring, is located in the same segment of the ring as most ULXs. These powerful H/ULXs appear to be coincident with giant HII region complexes, young star clusters, and radio and midinfrared hot-spots: all strong indicators of recent massive star formation. The X-ray spectra show that H/ULXs have similar properties as those of the most luminous ULXs found in the nearest starbursts and galaxy mergers such as the Antennae galaxies and M82. The close association between the $\mathrm{X}$-ray sources and the starbursting ring strongly suggests that the H/ULXs are intimately associated with the production and rapid evolution of short-lived massive stars. The observations represent the most extreme X-ray luminosities discovered to date associated with star-forming regions - rivaling the $\mathrm{X}$-ray luminosities usually associated with active galactic nuclei.

Subject headings: galaxies: individual (VV 784, Cartwheel, ESO 350-G 040) - galaxies: interactions — galaxies: active — galaxies: starburst - X-rays: galaxies
\end{abstract}

\section{INTRODUCTION}

The high resolution capabilities of the Chandra have led to the discovery of a large population of extraordinarily X-ray luminous point-like non-nucleus sources in many nearby galaxies (e.g., Fabianno, Zezas, \& Murray 2001; Colbert et al. 2003). These so called ultraluminous X-ray sources (ULXs; Makishima et al. 2000) can have apparent isotropic broad-band X-ray luminosities of $L_{0.5-10 \mathrm{keV}}$ : hundreds of times the Eddington limit for a neutron star or a stellar mass black hole. Although rarely present in normal galaxies, very luminous ULXs with $L_{0.5-10 \mathrm{keV}} \approx 3 \times 10^{39} \mathrm{ergs} \mathrm{s}^{-1}$ are often found in starbursts and IR-luminous galaxy mergers. For example, in both the Antennae galaxies and the luminous infrared (IR) galaxy merger NGC 3256, more than half a dozen such luminous ULXs are detected (Fabianno et al. 2001; Lira et al. 2002), and numerous less luminous pointlike sources are spread over the merging disks (Zezas et al. 2002). The Chandra observations of the Cartwheel galaxy ${ }^{4}$ presented here show an unusually large number (>20) of high-luminosity ULXs, most of which appear highly correlated with the narrow, young, outer starburst ring.

Hyperluminous X-ray sources (HLXs, $L_{0.5-10 \mathrm{keV}} \gtrsim 10^{41} \mathrm{ergs} \mathrm{s}^{-1}$, Matsumoto et al. 2001; Kaaret et al. 2001) are intriguing as they are apparently more luminous than the entire X-ray luminosity of a

\footnotetext{
1 University of Massachusetts, Department of Astronomy, LGRT-B 619E, 710 North Pleasant Street, Amherst, MA 010039305

2 SIRTF Science Center, Caltech, MS 220-6, 1200 E. California Blvd.,Pasadena, CA 91125

3 Space Telescope Science Institute, 3700 San Martin Drive, Baltimore, MD 21218

${ }^{4} H_{0}=75 \mathrm{~km} \mathrm{~s}^{-1} \mathrm{Mpc}^{-1}\left(c z=9090 \mathrm{~km} \mathrm{~s}^{-1}, d_{\mathrm{L}}=122 \mathrm{Mpc}\right)$ is used in this paper
}

normal galaxy - with luminosities approaching that of luminous active galactic nuclei (AGNs). H/ULXs appear variable (Fabianno et al. 2003) - their peak luminosity often changing by more than an order of magnitude within months to years (Matsumoto et al. 2001; Strickland et al. 2001). Here, we report an extraordinary HLX, as well as more than a dozen ULXs, located within and along the same portion of the active star-forming outer ring of the Cartwheel.

The Cartwheel galaxy has been studied in the radio (Higdon 1996), IR (Marcum, Appleton, \& Higdon 1992; Charmandaris et al. 1999), optical (Hidgon 1995; Struck et al. 1996; Amram et al. 1998), and X-rays (Wolter, Trinchieri, \& Iovino 1999). The crisp outer ring is believed to have been created as stars formed in radiallyexpanding density waves caused by a companion ("intruder") galaxy plunging through the center of a gasrich disk (see Lynds \& Toomre 1976; Toomre 1977; and review by Appleton \& Struck 1996). The outer ring appears to have propagated into a low-metallicity region of the disk (Fosbury \& Hawarden 1977) where it has triggered recent active star formation.

Both $\mathrm{H} \alpha$ and radio continuum observations (Higdon 1995, 1996) indicate that the dominant star-forming sites are in the outer ring of the southern quadrant, which constitutes about $80 \%$ of the total emission from the entire galaxy. Chandra observations discussed here clearly show that the dominant X-ray emission originates in the same starbursting southern ring quadrant.

\section{IMAGING ANALYSIS \& SPECTRAL FITTING}

The Chandra data analysis of the Cartwheel is part of a systematical, uniform, archival study of nearly 20 interacting/merging galaxies. The Cartwheel was observed by ACIS-S (OBSID: 2019, PI: A. Wolter) with a $75 \mathrm{ksec}$ exposure from 2001 May 26 to 27. Data calibration was 
performed with CIAO v2.3 using the latest calibration database. Events files of level one were obtained from the Chandra archive, and were corrected for the aspect offset before processing into level two. Bad pixels and background flares as well as streaks were removed, and final clean maps were created. Further data analysis and image processing were done in IDL. This includes source detection, images of individual bands, and image smoothing (see Wang, Chaves, \& Irwin 2003). We also followed various CIAO threads to extract the X-ray spectra for point-like and extended sources. Spectral fitting was done with XSPEC. We also ran the timing analysis, but none of the point-like sources showed detectable variability within the observing time frame.

Fig. 1a shows broad-band X-ray contours overlaid on an HST/WFPC2 B-band image. Almost all the Xray emission in the Cartwheel originates from point-like sources within the southern quadrant of the outer ring. The sources are nearly coincident with the strong $\mathrm{H} \alpha$, radio continuum emission and blue super-star clusters (SSCs). Other portions of the outer ring show little Xray emission except where exceptionally bright SSCs are seen. We label all the point-like sources in the immediate surroundings of the Cartwheel in Fig. 1b and detail them in Table 1.

The companion galaxy G1 (spiral) contains 6 point-like $\mathrm{X}$-ray sources, and the early-type spiral G2 is seen as a fainter diffuse source (Fig 1). The farthest companion galaxy G3 is also significantly detected, with one ULX in the eastern edge of its disk. In addition, a faint, diffuse X-ray envelope which includes the Cartwheel, G1 and G2 is marginally detected.

The absence of any point-like X-ray source in the nuclear region of the Cartwheel rules out the existence of AGN. Although rather complex optical structures (Struck et al. 1996), and prominent mid-IR emission (Charmandaris et al. 1999) in the inner disk/ring, the X-ray emission from Cartwheel's inner disk, including the nucleus, is extremely weak.

Table 2 summarizes our spectral fitting. For the strongest, source 11, both an absorbed Raymond-Smith (RS) and Mekal thermal plasma models failed to yield the acceptable fit, giving an unrealistically high temperature, $\gtrsim 10 \mathrm{keV}$, for the plasma. The absorbed power-law (PO) and multicolor accretion disk (MCD) models are both acceptable, but the data show excess emission features around $1.4 \mathrm{keV}$. This appears to be similar to the line features ( $\mathrm{Mg} X I$ and $\mathrm{Mg} X I I I)$ observed in a few ULXs in the Antennae (Zezas et al. 2002). We thus modeled the spectrum with an additional narrow Gaussian (Gau) component to the PO (Fig. 2a) and MCD models. Both can give improved fit to the data.

Assuming isotropic emission, the absorption-corrected hard and broad-band X-ray luminosities $L_{2-10 \mathrm{keV}}$ and $L_{0.5-10 \mathrm{keV}}$ of source 11 are $\sim 0.6-0.9$ and 0.9 $1.3 \times 10^{41} \mathrm{ergs} \mathrm{s}^{-1}$ respectively. Thus, this source is an HLX, and could have a total luminosity $L_{0.05-100 \mathrm{keV}}$ as large as $\sim 5.0 \times 10^{41} \mathrm{ergs} \mathrm{s}^{-1}$. In comparison, ROSAT data suggest an intrinsic $L_{0.5-5 \mathrm{keV}} \sim 2.3 \times 10^{41} \mathrm{ergs} \mathrm{s}^{-1}$ for the detected outer ring assuming $N_{\mathrm{H}}=2 \times 10^{21} \mathrm{~cm}^{-2}$ (Wolter et al. 1999), and most of the emission detected by ROSAT is presumably from source 11 .

The whole southern ring, including diffuse emission and all the sources, taken together, can be fit well with an absorbed $\mathrm{PO}+\mathrm{RS}$ model. So can the diffuse emission of the entire system (Fig. 2b,c, Table 2). The un-absorbed hard and broad-band X-ray luminosities of the south ring are 1.1 and $3.8 \times 10^{41} \mathrm{ergs} \mathrm{s}^{-1}$. And the total luminosity $L_{0.05-100 \mathrm{keV}}$ is as large as $1.2 \times 10^{42} \mathrm{ergs} \mathrm{s}^{-1}$. Therefore, on average, all detected point-like sources in the south ring would truly be ULXs, more luminous than the most luminous ULXs $\left(L_{0.5-10 \mathrm{keV}} \sim 6 \times 10^{39} \mathrm{ergs} \mathrm{s}^{-1}\right)$ detected in the Antennae. Although detailed spectral analysis is difficult for each of these point-like sources owing to the limited count statistics $(\sim 100$ photons $)$, we can roughly estimate the X-ray flux according to their count rates. For instance, each of the double-ULX (sources 2 \& 3) in the northwest end of the south ring has a count rate only 3 times smaller than that of the HLX. Both of the double-ULX (sources 15 \& 17) southeast of the HLX also have count rates only a factor of 5 lower than that of the HLX. Thus, in combination, these 4 ULXs might have X-ray luminosity comparable to that of the HLX. The faintest sources detected have a luminosity of $L_{0.5-10 \mathrm{keV}} \sim 3 \times 10^{39} \mathrm{ergs} \mathrm{s}^{-1}$.

A point-like source $31, \sim 10 \mathrm{kpc}$ north of G2, is likely a background galaxy or AGN as it has a faint optical counterpart in the HST image. Limited spectrum can be fit by an absorbed PO with a photon index $\sim 1.9$, but a much less absorption column density $N_{\mathrm{H}} \sim 0.5 \times$ $10^{21} \mathrm{~cm}^{-2}$.

\section{ACTIVE STAR-FORMING RING KNOTS AND THE $\mathrm{H} / \mathrm{ULXS}$}

Most H/ULXs are located within the portion of the Cartwheel ring which is experiencing the most powerful current star formation. Few ULXs discovered so far have prominent optical counterparts (e.g., Immler et al. 2003; Wu et al. 2001), but almost all H/ULXs in the Cartwheel are closely associated with giant complexes of HII regions and blue SSCs (Fig. 1a).

Although the HLX lies within $\sim 10^{\prime \prime}$ of the strongest $\mathrm{H} \alpha$ knot, CW-17, which is also the hot-spot in radio and mid-IR (Higdon 1995; Charmandaris et al. 1999), it is not coincident with this strongest starburst. Rather the HLX lies closer $\left(1.7^{\prime \prime} \sim 1 \mathrm{kpc}\right)$ to the fainter $\mathrm{H} \alpha$ feature CW-20. In contrast, CW-17 coincides with a much softer (and fainter) ULX source 12. Hence the HLX defies an absolute one-to-one correspondence with an optical knot, although it lies within the arc of bright $\mathrm{H} \alpha$ and radio emission which characterizes this portion of the ring.

We also give cross-identifications from the position match between $\mathrm{H} / \mathrm{ULXs}$ and $\mathrm{H} \alpha$ knots in Table 1. All 5 strongest $\mathrm{H} \alpha$ knots (CW-14, 15, 17, 24, 25) with $\mathrm{H} \alpha$ luminosity $\left.\gtrsim 1.3 \times 10^{41} \mathrm{ergs} \mathrm{s}^{-1}\right), 20$ times more luminous than 30 Doradus (Wang 1999), are coincident with the H/ULXs within $1^{\prime \prime} .7$. Moreover, except three ULXs (sources 10, 20, \& 21) interior to, and others (e.g., source 16 and those near G1, G2) outside of the outer ring, all $\mathrm{H} /$ ULXs seem to have spatial correspondence with the ring of giant HII region complex. Nevertheless, a number of fairly strong HII regions do not have corresponding $\mathrm{X}$-ray sources.

The evidence that, 1) HLX lies close to an HII region, 2) all five of the strongest $\mathrm{H} \alpha$ knots have corresponding ULXs, 3) the large number of matches between $\mathrm{H} \alpha$ knots and other ULXs in the narrowly defined ring, leads to the conclusion that H/ULXs appear to be directly linked to 
the production and evolution of the short-lived massive stars in the Cartwheel.

\section{NATURE OF H/ULXS}

The Cartwheel is the record holder in hosting both the HLX and the largest number of the most luminous ULXs in one galaxy. It has been argued on observational and theoretical grounds (Appleton \& Struck-Marcell 1996; Bransford et al. 1998) that the triggering of newlyformed stars in ring galaxies occurs approximately simultaneously as the wave propagates out through the diskthe outer ring representing the most recently formed stars, with representative ages $<10^{7}$ yrs. In this picture, the ring represents the outermost progress of a wave that began at the disk-center some 300 Myrs previously, created by the central perturbation of the intruder, either G3 or G1 (Mihos \& Hernquist 1994; Higdon 1996; Struck et al. 1996). The striking similarity between the X-ray source distribution and the young SSCs and HII regions suggests a strong causal connection between them. The lack of radial spread in the ring X-ray sources (with the exception of the three interior sources) indicates that, like the star-forming ring, the X-ray sources are linked to the active star formation episode and their young $(<$ 10 Myrs) ages.

The two most likely sources of X-ray emission associated with massive young star-forming regions are probably supernovae (SNe) or extremely young SN remnants (SNRs) and the high-mass X-ray binaries (HMXBs). We can almost rule-out low-mass X-ray binaries (LMXBs) to be the significant sources for $\mathrm{H} / \mathrm{ULXs}$ along the Cartwheel narrow ring, although intermediatemass black holes (IMBHs, see review by Miller \& Colbert 2003) are likely viable. It is conceivable that LMXBs and/or background sources could be responsible for the three ULXs interior to the ring. Three "ULXs" outside the Cartwheel with faint optical counterparts are likely background galaxies. We restrict ourselves here to the majority of $\mathrm{H} / \mathrm{ULX}$ s in the ring.

The interaction between the expanding SN ejecta and the dense circumstellar medium of the progenitor massive star can produce high X-ray luminosity $\sim 10^{40} \mathrm{ergs} \mathrm{s}^{-1}$ (e.g., Pooley et al. 2002). Therefore, such young "SNRs" are not like classical Cas-A and are extremely bright with hard X-ray spectra. Some ULXs seem close to being resolved by Chandra at $0.5^{\prime \prime}(300 \mathrm{pc})$ resolution, suggesting that each ULX may be a composite of several individual luminous SNRs. If we adopt a SN rate for the outer ring of $0.1-1 \mathrm{yr}^{-1}$ derived from the non-thermal radio continuum (Higdon 1996), and a minimum age for the ring HII regions of 1 Myrs, then we would predict a few times $10^{5}$ SNRs created over this period. Assuming that the X-ray phase of young SNRs is very short-lived $\left(\lesssim 10^{2} \mathrm{yrs}\right)$, then we might predict a few tens of young SNRs in the outer ring based on the radio flux alone. The individual ULXs in the ring could plausibly be collections of several such young SNRs. ULXs 6-8, 12-15 \& 17 (Fig. 1b) may be in this category, being closely associated with radio hotspots. Source 21, which lies just inside the outer ring, has no $\mathrm{H} \alpha$ emission, but has radio emission, may also be associated with SNRs.

In contrast, the HLX has only weak extended emission in both $\mathrm{H} \alpha$ and radio continuum. Its extreme X-ray luminosity does not seem to favor young SNRs unless they are unusually bright. Spectral fitting (Fig. 2a) also does not support a SNR scenario for the HLX, given both absorbed PO and MCD models could fit the data, whereas the thermal plasma models failed. This source may be the best candidate for a HMXB and/or IMBH since the formation of massive black hole in dense star clusters is possible (e.g., Rasio, Freitag, \& Gürkan 2003).

\section{REFERENCES}

Appleton, P.N., \& Struck-Marcell, C. 1996, Fund. of Cosmic Phy., 16,111

Amram, P., Mendes de Oliveira, C., Boulesteix, J., \& Balkowski, C. $1998, A \& A, 330,881$

Bransford, M., Appleton, P. N., Marston, A. P. \& Charmandaris, V. 1998, AJ, 116, 2757

Charmandaris, V. et al. 1999, A\&A, 341, 69

Colbert, E.J.M., et al. 2003, ApJ, submitted (astro-ph/0305476

Fabbiano, G., Zezas, A., \& Murray, S.S. 2001, ApJ, 554, 1035

Fabbiano, G., Zezas, A., King, A.R. et al. 2003, ApJ, 584, L5

Fosbury, R.A.E., \& Hawarden, T.G. 1977, MNRAS, 178, 473

Higdon, J.L. 1995, ApJ, 455, 524

Higdon, J.L. 1996, ApJ, 467, 241

Immler, S., Wang, Q.D., Leonard, D.C., \& Schlegel, E.M. 2003, ApJ, in press

Kaaret, P., et al. 2001, MNRAS, 321, L29

Lira, P., et al. 2002, MNRAS, 330, 259

Lynds, R., \& Toomre, A. 1976, ApJ, 209, 382

Makishima, K., et al. 2000, ApJ, 535, 632

Marcum, P.M., Appleton, P.N., \& Higdon, J.L. 1992, ApJ, 399, 57
Matsumoto, H., et al. 2001, ApJ, 547, L25

Mihos, J.C., \& Hernquist, L. 1994, ApJ, 437, 611

Miller, M.C., \& Colbert, E.J.M. 2003, astro-ph/0308402

Pooley, D., Lewin, W.H.G., Fox, D.W. et al. 2002, ApJ, 572, 932

Rasio, F.A., Freitag, M., \& Gürkan, M.A. 2003, astro-ph/0304038

Strickland, D.K., Colbert, E.J.M., Heckman, T.M. et al. 2001, ApJ, 560,707

Struck, C., Appleton, P.N., Borne, K.D., \& Lucas, R. 1996, AJ, 112,1868

Toomre, A. 1977, in IAU Symp. 79, ed. M.S. Longair \& J. Einasto (Dordrecht: Reidel), 109

Wang, Q.D. 1999, ApJ, 510, L139

Wang, Q.D., Chaves, T., \& Irwin, J.A. 2003, ApJ, in press (astro-ph/0308150)

Wolter, A., Trinchieri, G., \& Iovino, A. 1999, A\&A, 342, 41

Wu, H., Xue, S.J., Xia, X.Y., Deng, Z.G., Mao, S. 2002, ApJ, 576, 738

Zezas, A., Fabbiano, G., Rots, A.H., \& Murray, S.S. 2002, ApJS, 142,239 
Table 1. Point-like X-ray Sources (H/UlXs) in the Cartwheel Galaxy SYSTEM

\begin{tabular}{|c|c|c|c|c|}
\hline No. & CXO Name & $\mathrm{CR}\left(\mathrm{cts} \mathrm{ks}^{-1}\right)$ & $\mathrm{H} \alpha \quad \mathrm{ID}^{\mathrm{a}}$ & Offset $\left({ }^{\prime \prime}\right)$ \\
\hline 1 & J003737.56-334342.7 & $0.13 \pm 0.05$ & - & - \\
\hline 2 & J003737.59-334257.3 & $2.11 \pm 0.21$ & CW-26 & 2.5 \\
\hline 3 & J003737.61-334255.8 & $1.91 \pm 0.19$ & $\mathrm{CW}-27$ & 3.1 \\
\hline 4 & J003737.87-334253.6 & $0.16 \pm 0.06$ & - & - \\
\hline 5 & J003738.36-334309.3 & $0.24 \pm 0.07$ & CW-25 & 3.0 \\
\hline 6 & J003738.75-334316.7 & $1.28 \pm 0.15$ & $\mathrm{CW}-24$ & 1.4 \\
\hline 7 & J003738.82-334319.1 & $0.72 \pm 0.12$ & $\mathrm{CW}-23$ & 0.7 \\
\hline 8 & J003738.97-334317.5 & $0.16 \pm 0.06$ & - & - \\
\hline 9 & J003739.16-334230.9 & $0.60 \pm 0.10$ & CW-29 & 1.3 \\
\hline 10 & J003739.22-334250.6 & $0.96 \pm 0.13$ & - & - \\
\hline 11 & J003739.40-334323.7 & $5.75 \pm 0.31$ & $\mathrm{CW}-20$ & 1.7 \\
\hline 12 & J003740.26-334327.5 & $0.28 \pm 0.07$ & CW-17 & 1.4 \\
\hline 13 & J003740.46-334325.4 & $0.24 \pm 0.07$ & - & - \\
\hline 14 & J003740.74-334330.7 & $0.35 \pm 0.08$ & CW-15 & 0.5 \\
\hline 15 & J003740.88-334331.3 & $1.04 \pm 0.13$ & CW-14 & 1.7 \\
\hline 16 & J003741.06-334221.9 & $0.31 \pm 0.08$ & - & - \\
\hline 17 & J003741.09-334332.3 & $1.26 \pm 0.16$ & CW-12 & 1.6 \\
\hline 18 & J003741.73-334235.7 & $0.15 \pm 0.06$ & CW-3/4 & $2.3 / 2.7$ \\
\hline 19 & J003742.01-334326.8 & $0.20 \pm 0.07$ & CW-10/9 & $1.8 / 0.7$ \\
\hline 20 & J003742.15-334314.2 & $0.31 \pm 0.09$ & - & - \\
\hline 21 & J003742.50-334304.5 & $0.33 \pm 0.08$ & - & - \\
\hline 22 & J003742.80-334212.8 & $0.67 \pm 0.12$ & - & - \\
\hline 23 & J003742.87-334210.2 & $0.32 \pm 0.08$ & - & - \\
\hline 24 & J003742.96-334204.6 & $0.69 \pm 0.12$ & - & - \\
\hline 25 & J003743.02-334206.3 & $0.84 \pm 0.13$ & - & - \\
\hline 26 & J003743.13-334143.3 & $0.23 \pm 0.07$ & - & - \\
\hline 27 & J003743.14-334204.4 & $1.13 \pm 0.15$ & - & - \\
\hline 28 & J003743.70-334147.6 & $0.25 \pm 0.07$ & - & - \\
\hline 29 & J003743.87-334210.2 & $0.67 \pm 0.11$ & - & - \\
\hline 30 & J003745.32-334229.0 & $1.13 \pm 0.15$ & - & - \\
\hline 31 & J003745.63-334152.3 & $4.91 \pm 0.33$ & - & - \\
\hline
\end{tabular}

${ }^{a} \mathrm{H} \alpha$ knots as identified by Higdon (1995) with a point-spread function of $1 .{ }^{\prime \prime} 7$ FWHM.

Table 2. Spectral Fitting and Measurements of the Cartwheel

\begin{tabular}{|c|c|c|c|c|c|c|}
\hline Source & $\begin{array}{c}N_{\mathrm{H}} \\
10^{21} \mathrm{~cm}^{-2}\end{array}$ & $\begin{array}{c}\text { Model Parameters }{ }^{\mathrm{a}} \\
\Gamma, \mathrm{T}^{\mathrm{b}}\end{array}$ & $\chi^{2} /$ d.o.f. & $L_{2-10 \mathrm{keV}}$ & $\begin{array}{c}L_{0.5-10 \mathrm{keV}} \\
10^{41} \mathrm{ergs} \mathrm{s}^{-1}\end{array}$ & $L_{0.05-100 \mathrm{keV}}$ \\
\hline HLX & $3.5 \pm .7$ & $1.6 \pm .2, . .(\mathrm{PO})$ & $17.5 / 21$ & 0.8 & 1.3 & 4.1 \\
\hline HLX & $2.0 \pm .5$ &., $1.5 \pm .2(\mathrm{MCD})$ & $16.4 / 21$ & 0.6 & 0.9 & 1.0 \\
\hline HLX & $2.6 \pm .9$ & $1.4 \pm .2,1.4 \pm .1(\mathrm{PO}+\mathrm{Gau})$ & $11.2 / 18$ & 0.9 & 1.2 & 5.5 \\
\hline HLX & $1.6 \pm .5$ &.., $1.7 \pm .4 / 1.4 \pm .1(\mathrm{MCD}+\mathrm{Gau})$ & $9.5 / 18$ & 0.6 & 0.9 & 1.0 \\
\hline South Ring & $3.7 \pm 1.9$ & $2.2 \pm .3,0.18 \pm .02(\mathrm{PO}+\mathrm{RS})$ & $22.0 / 23$ & 1.1 & 3.8 & 12.5 \\
\hline All ${ }^{\mathrm{C}}$ & $1.8 \pm 2.6$ & $2.3 \pm .9,0.23 \pm 0.15(\mathrm{PO}+\mathrm{RS})$ & $6.2 / 12$ & .24 & .53 & 2.6 \\
\hline
\end{tabular}


models, respectively.

${ }^{\mathrm{b}} \Gamma$ is the photon index, various temperature $\mathrm{T}$ is in units of $\mathrm{keV}$.

cThe diffuse emission from Cartwheel, G1 and G2 and between them. But all point-like sources are excluded.

FIG. 1.- (a) Broad-band X-ray contours overlaid on the HST/WFPC2 optical image. The lowest contours are 0.0345 , 0.0431 cts/pixel (pixel $\sim 0.5^{\prime \prime}$ ), and then increase successively by a factor of 2 . (b) Soft $(0.3-1.5 \mathrm{keV}$ ) X-ray image overlaid with the hard X-ray contours $(1.5-7 \mathrm{keV})$ with sources labeled. Companion galaxies G1 and G2 are labeled too. The third companion galaxy G3 is $\sim 3^{\prime}$ in northeast, outside the region shown in the figure. 
FIG. 2. - The extracted X-ray spectra of: (a) The HLX and fit with the absorbed power-law (PO) plus Gaussian line features at 1.9 keV (top). (b) The entire ring of southern quadrant (middle). (c) The diffuse emission of the entire system with point-like sources subtgigcted (bottom). The fit in (b) and (c) are the absorbed PO plus Raymond-Smith thermal plasma models (Table 2).

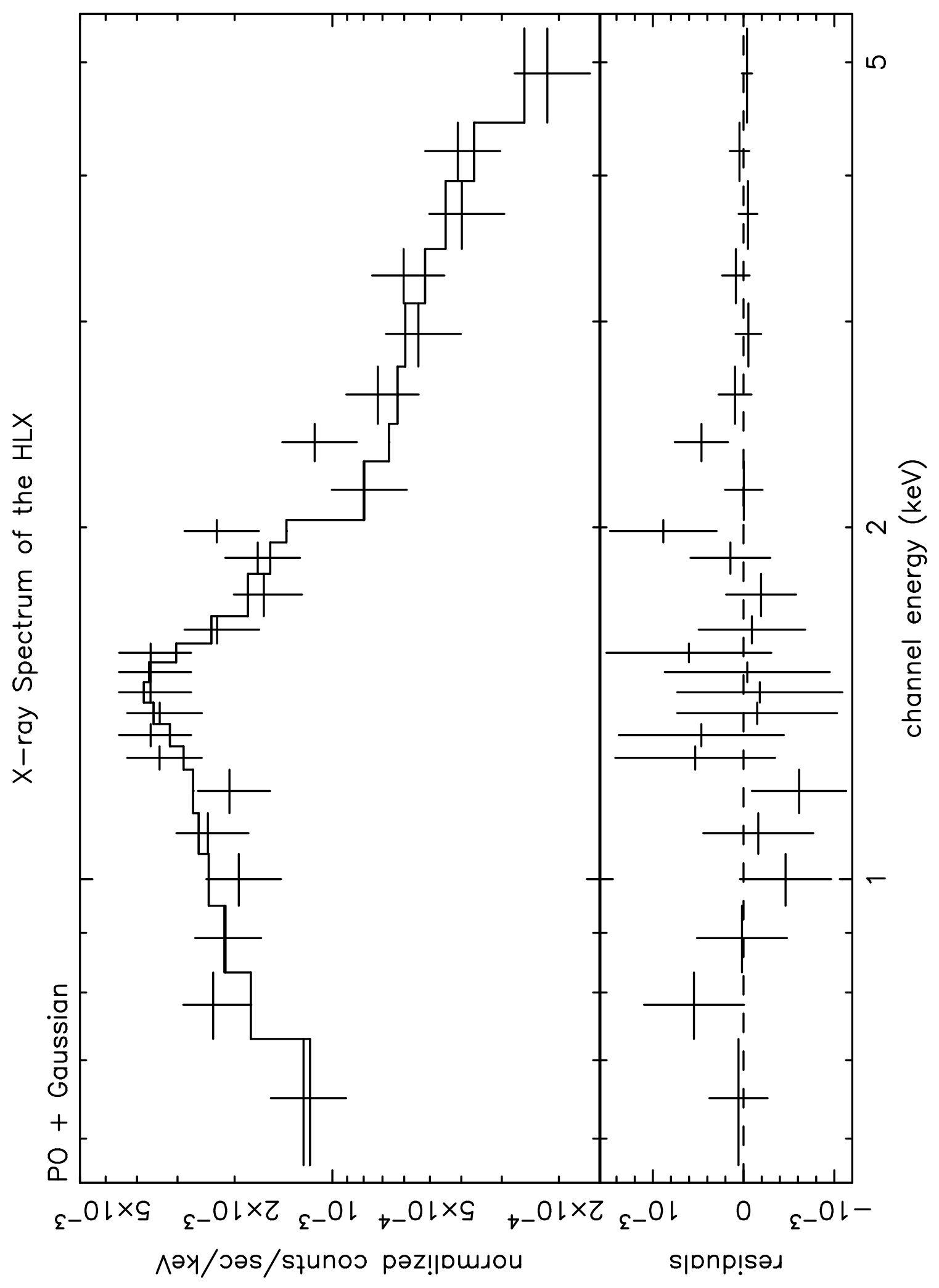




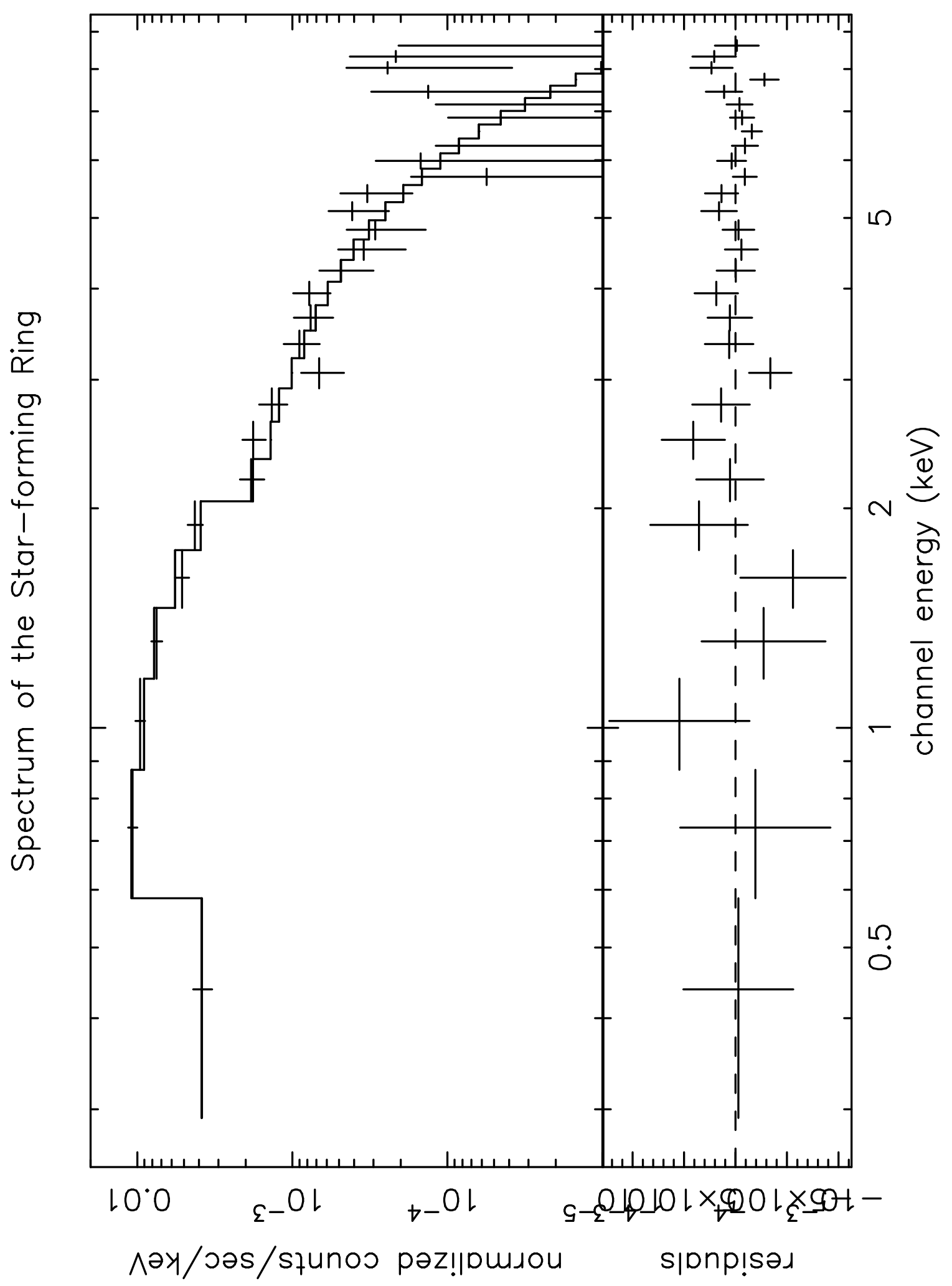




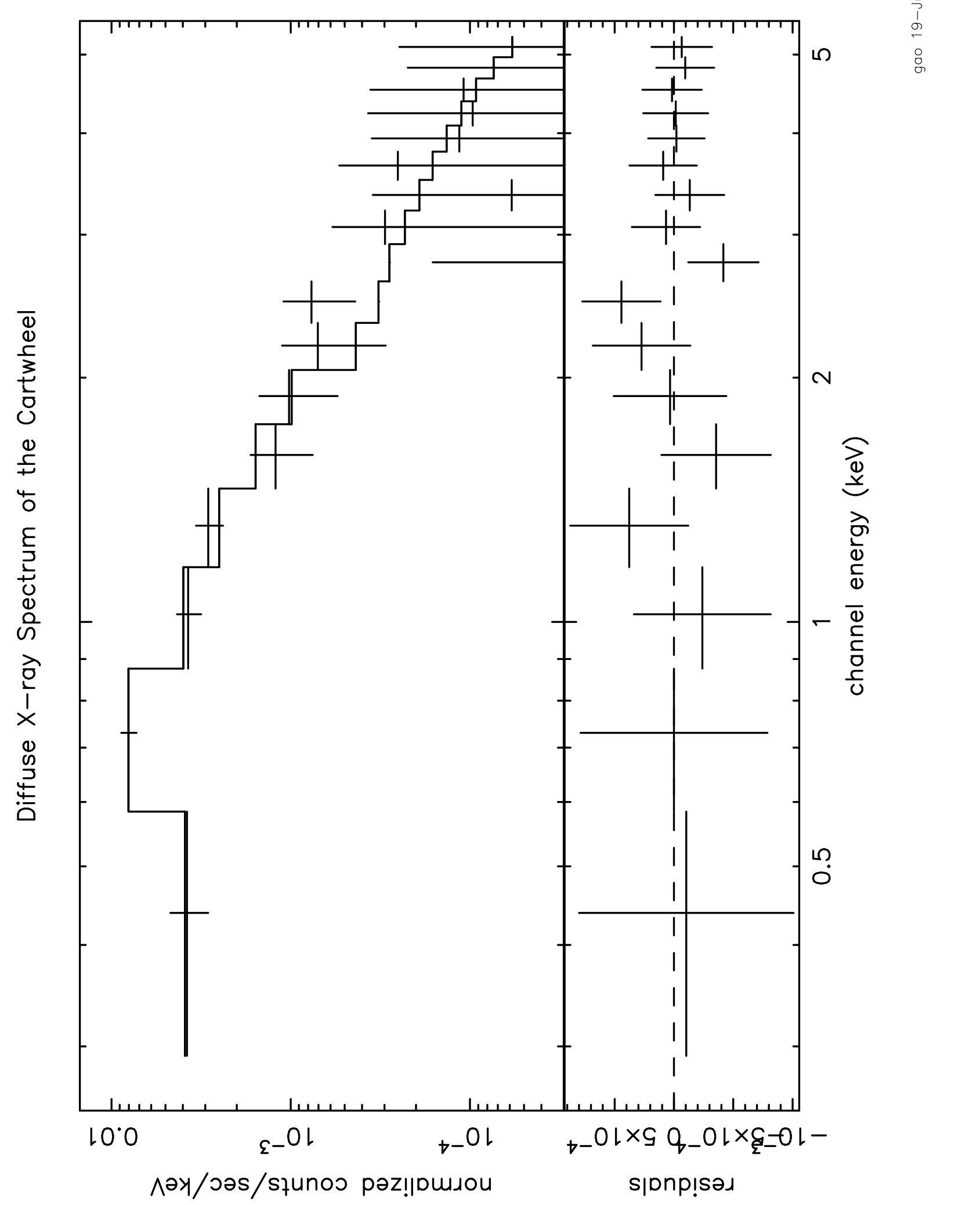


This figure "fla.gif" is available in "gif" format from: http://arXiv.org/ps/astro-ph/0309253v1 
This figure "flb.gif" is available in "gif" format from: http://arXiv.org/ps/astro-ph/0309253v1 